\title{
Presencia de una Educación Ambiental basada en conocimiento, actitudes y prácticas en la enseñanza de las ciencias naturales en establecimientos municipales de la ciudad de Los Ángeles, Chile
} Presence of an environmental education based on knowledge, attitudes, and practices in
the teaching of natural sciences in municipal establishments of Los Angeles, Chile

\author{
Laura Beatriz Torres Rivera ${ }^{a}$, Juan Eloy Benavides Peña ${ }^{b}$, \\ Catalina José Latoja Volloutac ${ }^{c}$ Elissette Rafaela Novoa Contreras ${ }^{d}$ \\ ${ }^{a}$ Escuela de Educación, Campus Los Ángeles, Universidad de Concepción. \\ Correo electrónico: latorres@udec.cl \\ ${ }^{\mathrm{b}}$ Escuela G-882, Las Quintas. \\ Correo electrónico: juabenavides@udec.cl \\ ${ }^{\mathrm{c}}$ Colegio Alemán, Los Ángeles. \\ Correo electrónico: catalatoja@udec.cl \\ ${ }^{\mathrm{d}}$ Programa Magister Didáctica de las Matemáticas, Universidad de Concepción \\ Correo electrónico: elissettenovoa@udec.cl
}

\begin{abstract}
En Chile la Educación Ambiental se contempla desde un lineamiento curricular transversal e interdisciplinario y, según el Ministerio de Educación, es el docente quien debe incluirla a través de su labor pedagógica. Esta investigación evaluó la presencia de los ejes principales de la Educación Ambiental: conocimientos, actitudes y práctica, en docentes que imparten la asignatura de Ciencias Naturales en establecimientos municipales de la ciudad de Los Ángeles, Chile. La información se obtuvo por medio de una encuesta con escalamiento de tipo Likert, la cual fue validada y sometida a pilotaje. Los resultados evidenciaron que los docentes no están contemplando de manera cohesionada los tres ejes principales de la EA en la asignatura de Ciencias Naturales, en consecuencia los alumnos de primer y segundo ciclo básico de la ciudad de Los Ángeles no están recibiendo satisfactoriamente la enseñanza de la EA, de acuerdo a los requerimientos del Ministerio de Educación.
\end{abstract}

Palabras clave: enseñanza, pedagogía básica, profesores.

\section{ABSTRACT}

In Chile, environmental education is contemplated based on cross-curricular and interdisciplinary guidelines, and, according to the Ministry of Education, it is the teacher who must incorporate it through his or her individual teaching work. This research evaluated the presence of the main axes of environmental education (knowledge, attitudes, and practice) in natural sciences teachers from municipal schools in the city of Los Angeles, Chile. The information was obtained through a survey with the scaling developed by Likert, which was validated and subjected to a control. The results showed that teachers are not cohesively contemplating the three main axes of environmental education in the natural sciences, and consequently pupils in first and second grade in the city of Los Angeles are not receiving satisfactory teaching in the area of environmental science according to the requirements of the Chilean Ministry of Education.

Keywords: teaching, primary education, teachers. 
Estudios Pedagógicos XLIII, N 3: 311-323, 2017

PRESENCIA DE UNA EDUCACIÓN AMBIENTAL BASADA EN CONOCIMIENTO, ACTITUDES Y PRÁCTICAS EN LA ENSEÑANZA DE LAS CIENCIAS NATURALES EN ESTABLECIMIENTOS MUNICIPALES DE LA CIUDAD...

\section{INTRODUCCIÓN}

Hoy en día, debido a los problemas ambientales que enfrenta la sociedad, la formación ambiental de la ciudadanía se ha transformado en una necesidad urgente; sin embargo, esta es larga y compleja, pues incluye conceptos, procedimientos y en especial, valores y actitudes (Aydim, 2012). Una forma de alcanzar lo anterior y junto con esto el anhelado desarrollo sustentable, es implementando una adecuada Educación Ambiental (EA) (Fith y Winter, 2007), la cual de acuerdo a lo establecido en la carta de Belgrado (Organización de las Naciones Unidas para la Educación, la Ciencia y la Cultura, 1975), tiene como meta formar una población mundial que tenga conciencia del medio ambiente y se interese por él y por los problemas vinculados.

A partir del establecimiento de esta meta, las distintas naciones han trabajado para incorporar la EA bajo una metodología formal en los procesos de enseñanza en educación primaria y secundaria (Fith y Winter, 2007; Blum, 2008; Yavetz, Goldman y Pe'er, 2009), algunos apoyados por la creación de oficinas gubernamentales que coordinan esta acción (Fith y Winter, 2007; Blum, 2008); así como, con una constante evaluación de la implementación de metodologías innovadoras en el curriculum (Fith y Winter, 2007). Pero, independiente de cómo se incorpora la EA en el curriculum escolar, hay un consenso en que ésta debe estar soportada por tres ejes o dimensiones: conocimiento, habilidades y actitudes (Lee y Willians, 2001).

En Chile, la EA está considerada como un Objetivo de Aprendizaje Transversal (OAT), de modo que se instaura en la educación desde un lineamiento curricular transversal a todas las disciplinas, teniendo como función la construcción de valores, conocimientos y relaciones que favorezcan a la naturaleza y sus recursos. Por tanto, es imprescindible que ésta integre todos los ámbitos educativos, tales como: su identidad, aprendizajes diarios, familia y comunidad, relaciones sociales, entorno físico para que así se adopte como una forma de vida para los alumnos y alumnas. Sin embargo, el cumplir con la transversalidad queda a criterio de cada docente (Cabezas, 1997), razón por la cual, al momento de enfrentar la EA es fundamental la calidad del profesor debido a que es el mediador entre lo que se diseña en el curriculum y el aprendizaje (López, 2001; Sureda-Negre, OliverTrobat, Catalán-Fernández y Comas-Forgas, 2014), así como, en el proceso de adquisición de conciencia ambiental por parte de los estudiantes (Sureda-Negrete et al., 2014).

Por lo tanto, la tarea del docente es imprescindible para la creación de conciencia ambiental y valores en sus alumnos y a fin de cuentas, en la sociedad, para así lograr el anhelado equilibrio entre la relación de las personas con su entorno social y natural (Ferreira, Ryan, Tilbury, 2007; Esa, 2010). Lo anterior, es destacado por Wilke (1995) quien menciona que se necesita un maestro con un alto nivel de integridad, que garantice además de los conocimientos necesarios, el desarrollo de habilidades y la formación de valores que hoy exige nuestra sociedad para el cuidado y conservación de nuestro entorno, debido a que, un profesor que tenga conocimientos y actitudes ambientales generará estudiantes ambientalmente alfabetizados (Tuncer et al., 2009). Recientemente, Quinn, Castera y Clement (2016) muestran a partir de un grupo de profesores titulados y en formación que hay una fuerte relación entre los que tienen visión antropocéntrica con actitudes negativas hacia el ambiente y entre los con visión no-antropocéntrica y antropomórfica con actitudes altamente positivas hacia la naturaleza. Por lo cual, es muy probable que la actitud del profesor hacia el ambiente se traspase inevitablemente hacia sus alumnos. 

ENSEÑANZA DE LAS CIENCIAS NATURALES EN ESTABLECIMIENTOS MUNICIPALES DE LA CIUDAD...

Lamentablemente, los datos sugieren que ha habido poco interés en incorpora la EA en la formación de profesores (Sureda-Negre et al., 2014) y como consecuencia la mayoría de los maestros no asumen la enseñanza de la EA durante su desempeño profesional. Lo anterior es ratificado por Kennelly, Taylor y Maxwell (2008) quienes sugieren que el desarrollo de contenidos de EA durante la formación de profesores es un soporte importante para que el futuro profesor realice enseñanza del ambiente en su escuela.

Sumado a lo anterior, Mc Pherson y Hernández (2001) afirman que es en la asignatura de ciencias en donde hay más posibilidad de desarrollar en los alumnos sentimientos de respeto hacia la naturaleza y conciencia en cuanto a la necesidad del cuidado y protección del ambiente. En consecuencia, la enseñanza de las Ciencias Naturales es el área de la enseñanza más factible para el desarrollo de la EA debido a que dentro de esta disciplina se busca que los alumnos exploren e investiguen el entorno natural con una actitud de respeto y responsabilidad por el medioambiente, que reconozcan los efectos de la actividad humana sobre éste, que aprendan las distintas posibilidades que ofrece el desarrollo productivo sustentable y que construyan una visión reflexiva y crítica frente a las medidas de protección existentes en la actualidad (Ministerio de Educación, 2012).

En concordancia con lo expuesto y según los objetivos que pretende alcanzar la EA y los OAT del Sistema Educativo Chileno, se hace necesario destacar que los ejes principales de la EA, conocimientos, actitudes y práctica, no pueden estar en desunión puesto que son el núcleo central de la EA, y si se pretende que un profesor de Ciencias Naturales forme ciudadanos ambientalmente responsables, cada uno de estos ejes debe formar parte integral de sus procesos de enseñanza.

De acuerdo con los antecedentes expuestos es importante realizar un diagnóstico de la presencia de los ejes principales de la EA (conocimientos, actitudes y práctica) en docentes que imparten la asignatura de Ciencias Naturales en establecimientos municipales de la ciudad de Los Ángeles. Entonces, si dentro del currículo está claramente establecido que la EA debe impartirse de manera transversal en todas las asignaturas, pero esto queda a criterio del profesor; entonces, se espera que el eje conocimientos de la EA sea el predominante en la enseñanza de las Ciencias Naturales.

\section{METODOLOGÍA}

Esta investigación se enmarca en un estudio cuantitativo, no experimental y de carácter transversal (Hernández, Fernández y Baptista, 2013). La variable independiente es la formación inicial docente y posteriores capacitaciones de los docentes y la dependiente corresponde a los tres ejes principales de la EA: conocimientos, actitudes y valores.

Por su parte, la población correspondió a 137 profesores de Educación General Básica de establecimientos educacionales municipales de la ciudad de Los Ángeles, que imparten la asignatura de Ciencias Naturales de Primer y Segundo ciclo básico, sin importar si éstos poseen formación generalista o especialización en el área. La muestra fue escogida de forma probabilística aleatoria simple y alcanzó a un número de 47 profesores/as, correspondiendo al $34 \%$ de la población.

La técnica que se utilizó para la recolección de datos fue una encuesta estructurada basada en la escala de Likert, en la cual hay una selección de ítem de acuerdo a niveles de respuesta (Vidal, 2005). La encuesta se constituyó con 12 aseveraciones que abordaron 
Estudios Pedagógicos XLIII, N 3: 311-323, 2017

PRESENCIA DE UNA EDUCACIÓN AMBIENTAL BASADA EN CONOCIMIENTO, ACTITUDES Y PRÁCTICAS EN LA ENSEÑANZA DE LAS CIENCIAS NATURALES EN ESTABLECIMIENTOS MUNICIPALES DE LA CIUDAD...

los tres ejes principales de la EA: conocimientos, actitudes y práctica, los cuales a su vez conformaron las dimensiones de ésta. La encuesta estuvo conformada por 6 preguntas de la dimensión conocimientos, 4 de la dimensión actitudes y 6 de la dimensión práctica, sin embargo, algunas de las preguntas aportaba a más de una dimensión. Los niveles de respuesta de la encuesta y los puntajes asignados a cada uno de estos se muestran en la tabla 1.

Tabla 1. Descripción de los niveles de respuesta de la encuesta y sus respectivos puntajes.

\begin{tabular}{|c|l|c|}
\hline Nivel de respuesta & \multicolumn{1}{|c|}{ Descripción } & Puntaje \\
\hline Siempre & $\begin{array}{l}\text { El docente encuestado presenta el criterio descrito en el Eje de } \\
\text { manera altamente satisfactoria. }\end{array}$ & 4 \\
\hline Casi siempre & $\begin{array}{l}\text { El docente encuestado presenta el criterio descrito en el Eje de } \\
\text { manera satisfactoria. }\end{array}$ & 3 \\
\hline A veces & $\begin{array}{l}\text { El docente encuestado presenta el criterio descrito en el Eje de } \\
\text { manera medianamente satisfactoria. }\end{array}$ & 2 \\
\hline Casi nunca & $\begin{array}{l}\text { El docente encuestado presenta el criterio descrito en el Eje de } \\
\text { manera limitado. }\end{array}$ & 0 \\
\hline Nunca & $\begin{array}{l}\text { El docente encuestado presenta el criterio descrito en el Eje de } \\
\text { manera insatisfactoria. }\end{array}$ & 1 \\
\hline
\end{tabular}

Se asignó un puntaje ideal y un puntaje mínimo aceptable para cada dimensión. El puntaje ideal se determinó considerando la sumatoria de todas las respuestas con el indicador "siempre", mientras que el puntaje mínimo aceptable se consideró como la sumatoria de todas las respuestas de la encuesta con el indicador "casi siempre". En la tabla 2 se aprecia el puntaje ideal y puntaje mínimo aceptable para cada dimensión con respecto al total de individuos encuestados, correspondiendo a 47 docentes.

Tabla 2. Puntaje ideal y mínimo aceptable por cada dimensión.

\begin{tabular}{|c|c|c|}
\hline Dimensión & Puntaje ideal & Puntaje mínimo aceptable \\
\hline Conocimientos & 1128 & 846 \\
\hline Actitudes & 752 & 564 \\
\hline Práctica & 1128 & 846 \\
\hline
\end{tabular}

Posterior a la elaboración de la encuesta, ésta se validó por un comité de expertos, quienes la analizaron desde el punto de vista de su constructo y pertinencia de las preguntas para cada dimensión. Luego, la encuesta validad fue sometida a pilotaje en un grupo de 17 profesores de dos establecimientos municipales, los cuales fueron excluidos en la selección de la muestra. 

ENSEÑANZA DE LAS CIENCIAS NATURALES EN ESTABLECIMIENTOS MUNICIPALES DE LA CIUDAD...

La encuesta se sometió al análisis de fiabilidad Alfa de Cronbach, el cual permitió medir los ítems en un mismo constructo para establecer si estos están altamente correlacionados (Welch y Comer, 1988). Como criterio general, George y Mallery (2003) establecieron que para evaluar los coeficientes de Alfa de Cronbach estos deben obtener un valor de fiabilidad superior a 0,8 para que el instrumento sea categorizado como bueno. El valor alcanzado por la encuesta en este análisis fue de 0,874 , verificándose así la fiabilidad del instrumento elaborado.

Con la finalidad de caracterizar la muestra para apoyar la interpretación de los resultados de la encuesta se incorporaron preguntas abiertas en donde cada profesor/a específico su título profesional, mención o especialidad, estudios y/o capacitaciones posteriores, años de ejercicio profesional, nivel de ejercicio profesional y género.

Los análisis de datos fueron realizados por dimensión a través del programa Excel XLSTAT. El análisis de normalidad realizado para cada dimensión de la encuesta se realizó aplicando las pruebas de Shapiro-Wilk, Anderson-Darling y Lilliefors. Al tener una distribución libre se le aplicó el Test de Rachas para una muestra y de esta manera se estableció que los datos están distribuidos al azar. .

\section{RESULTADOS}

\subsection{CARACTERIZACIÓN DE LA MUESTRA}

A partir de los resultados analizados, se desprende que la muestra presenta un $96 \%$ de docentes con título profesional en Educación General Básica, el otro porcentaje corresponde a profesionales que están impartiendo la asignatura de Ciencias Naturales con título profesional en Educación Diferencial y Educación Parvularia (gráfico 1a). Del total de la muestra, el 4\% de los docentes encuestados posee mención o especialidad en la asignatura de Ciencias Naturales, 75\% tiene mención o especialidad en otra área del aprendizaje y el resto de la muestra no posee mención o especialidad en ningún área (gráfico 1b).

Respecto a los estudios posteriores y/o capacitaciones que los docentes pueden optar a lo largo de su experiencia laboral, según la muestra extraída de la población de docentes que imparten la asignatura de Ciencias Naturales, sólo el 2\% del total ha realizado capacitaciones o cursos en esta área, además se aprecia que un $38 \%$ de la muestra examinada presenta estudios pertenecientes a otra área de aprendizaje o curricular. Cabe destacar que se presenta un alto porcentaje de docentes que no han aumentado sus competencias con estudios posteriores y/o capacitaciones (gráfico 1c). 
Estudios Pedagógicos XLIII, N 3: 311-323, 2017

PRESENCIA DE UNA EDUCACIÓN AMBIENTAL BASADA EN CONOCIMIENTO, ACTITUDES Y PRÁCTICAS EN LA ENSEÑANZA DE LAS CIENCIAS NATURALES EN ESTABLECIMIENTOS MUNICIPALES DE LA CIUDAD...

Gráfico 1. Caracterización de la muestra analizada a través de la encuesta, desglosándose a. Título profesional b. Mención o especialidad c. Estudios y/o capacitaciones posteriores d. Años de ejercicio profesional e. Nivel de ejercicio profesional f. Sexo.

a

口Educación General Básica 口Educación Diferencial

口Educación Parvularia

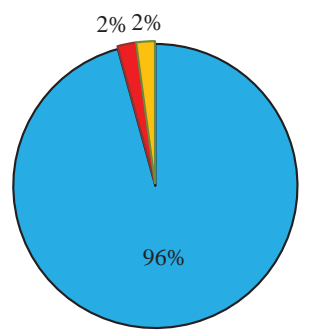

d

$\square 0$ a $10 \square 11$ a $20 \square 21$ a $30 \square 31$ a 40

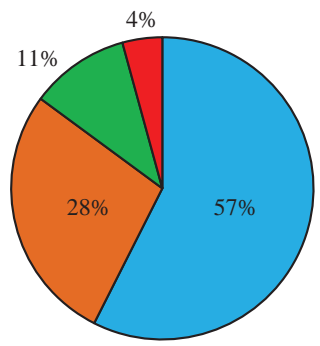

b
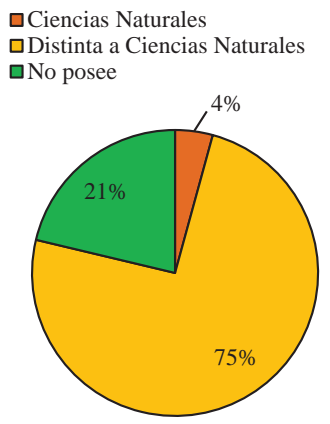

e

$\square 1^{\circ} \mathrm{CICLO}$ $\square 2^{\circ} \mathrm{CICLO}$ 口AMBOS CICLOS

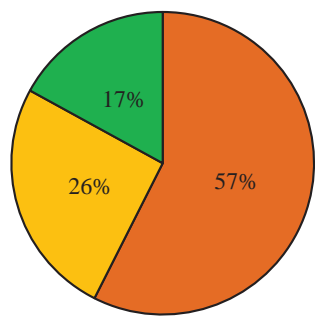

\section{C}
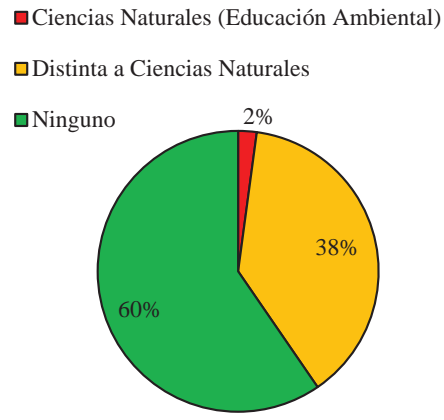

f

$\square$ Femenino $\quad \square$ Masculino

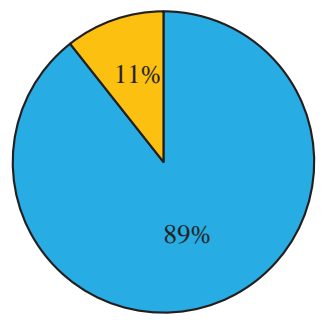

En la categoría de años de ejercicio profesional de los docentes encuestados, se aprecia un alto porcentaje en el rango mínimo determinado, entre 0 a 10 años, y un bajo porcentaje en el rango máximo determinado entre 31 a 40 años (gráfico 1d).

De acuerdo a los datos recopilados en el ítem nivel de ejercicio profesional, el 56\% de los docentes están realizando clases de Ciencias Naturales en el nivel de primer ciclo, un $26 \%$ están impartiendo clases en esta área en el nivel de segundo ciclo y además se aprecia que un bajo porcentaje de la muestra están realizando clases en ambos niveles educativos (gráfico 1e). Respecto al sexo de los docentes encuestados, se aprecia un dominio de profesores del género femenino sobre el masculino (gráfico 1f).

\subsection{PRESENCIA DE LOS EJES PRINCIPALES DE LA EDUCACIÓN AMBIENTAL}

Las respuestas proporcionadas por la muestra en la encuesta, muestra un total de 815 puntos de un máximo de 1128 puntos para el eje conocimientos. En este eje se consideró 846 puntos como el puntaje mínimo aceptable. Por tanto, la muestra encuestada no alcanzó el puntaje mínimo aceptable determinado para la dimensión (gráfico 2). 
Con respecto al eje actitudes, se obtuvo un total de 607 puntos de un máximo de 752 puntos. En este eje se consideró 564 puntos como el puntaje mínimo aceptable. Así, la muestra encuestada obtuvo 43 puntos por sobre el puntaje mínimo aceptable (gráfico 2).

Finalmente, en las respuestas proporcionadas por la muestra en el eje práctica, se obtuvo un total de 793 puntos de un máximo de 1128 puntos. En este se consideró 846 puntos como el puntaje mínimo aceptable; por lo cual, la muestra encuestada no alcanzó el puntaje mínimo aceptable determinado para la dimensión (gráfico 2).

Gráfico 2. Puntaje obtenido por los profesores en cada una de las dimensiones y su comparación con el puntaje ideal y mínimo aceptable.

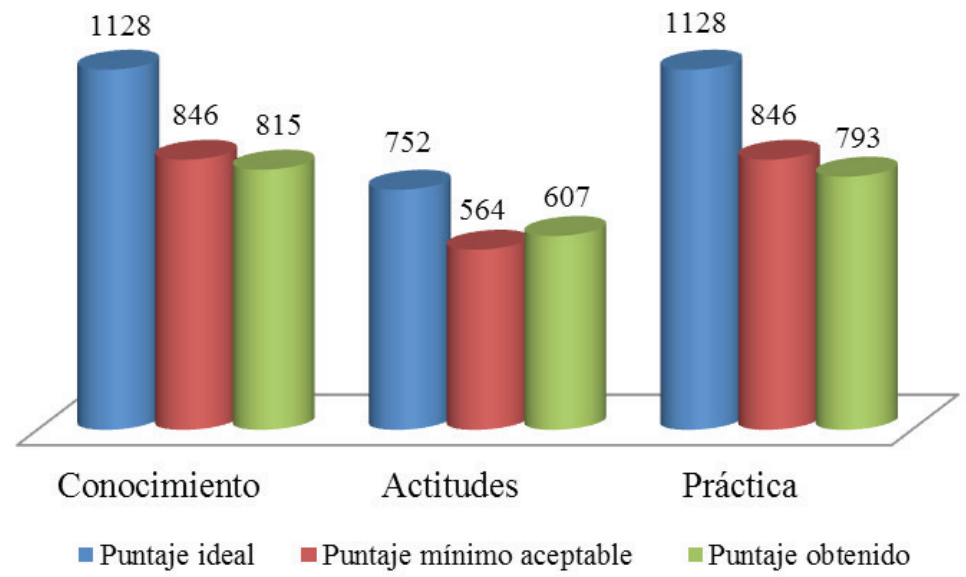

\section{DISCUSIÓN}

La agudización de los problemas ambientales ha obligado a plantear una nueva ética ambiental (Aydim, 2012), lo cual exige a los países formar ciudadanos conscientes de su entorno y con responsabilidad ambiental. La EA ha sido considerada como la herramienta fundamental para que todas las personas adquieran esa anhelada conciencia de su entorno y un estilo de vida acorde con el desarrollo sustentable (Firth y Winter, 2007), lo cual implica, realizar cambios en sus valores, conductas, estilos de vida y ampliar sus conocimientos para impulsar los procesos de prevención y resolución de los problemas ambientales presentes y futuros (González, 2003). Sin embargo, para que esto se pueda lograr es necesario que la EA esté cimentada sobre la base de conocimientos, actitudes y práctica, los cuales conforman sus tres ejes principales (Novo, 2003).

Entonces, si se considera a la EA el camino más confiable para cambiar el estilo de vida de las personas, los profesores pasan a ser actores relevantes de esta misión (López, 2001; Sureda-Negre et al., 2014), quienes deben transformarse en los agentes encargados de transmitir los conocimientos, actitudes y práctica y tener un rol activo en la formación ambiental de sus alumnos. Incluirla en la enseñanza constituye un trabajo permanente e interdisciplinario y que no todos los docentes le dedican el tiempo y el entusiasmo que esto 
Estudios Pedagógicos XLIII, N 3: 311-323, 2017

PRESENCIA DE UNA EDUCACIÓN AMBIENTAL BASADA EN CONOCIMIENTO, ACTITUDES Y PRÁCTICAS EN LA ENSEÑANZA DE LAS CIENCIAS NATURALES EN ESTABLECIMIENTOS MUNICIPALES DE LA CIUDAD...

conlleva. Es sabido que para realizar una EA efectiva los profesores necesitan altos niveles de confianza en sí mismos y en las materias que dictan (Çimen, Gökmen, Altunsoy, Ekici y Yilmaz, 2011), como también, conocimientos y actitudes pro ambientales (Tuncer et al., 2009).

Previo a esta investigación se esperaba que el eje conocimientos fuera el predominante en la enseñanza de las Ciencias Naturales, ya que algunos contenidos relacionados con la Educación Ambiental forman parte de los planes y programas del Ministerio de Educación y por ende, éstos deberían poseer los conocimientos básicos relacionados con las temáticas del medioambiente y su entorno. Sin embargo, este eje no alcanzó el puntaje mínimo aceptable, lo que demuestra que los docentes no se están informando ni actualizando constantemente sobre temáticas del medio ambiente. Una posible respuesta a los resultados obtenidos es que los docentes no estarían recibiendo una formación ambiental adecuada en su formación inicial docente que los prepare o los capacite para abordar éstas temáticas cuando están insertos en el sistema educativo.

Al revisar diversas mallas curriculares de la carrera de Educación General Básica con o sin especialidad de diferentes universidades chilenas, es poco frecuente encontrar asignaturas de EA dentro de éstas, sin embargo, en algunos casos esta temática es incorporada como asignatura electiva o complementaria. Shymansky y Kyle (1992) afirman que el primer paso para que la EA se incluya adecuadamente en el sistema educativo es introducirla en la formación inicial y permanente del profesorado, debido a que el docente sigue siendo, aún en las sociedades tecnológicamente más desarrolladas, el factor clave de la puesta en práctica de las innovaciones curriculares. Por tanto, la formación inicial docente proporciona una oportunidad para asegurar que los profesores reciban una buena formación en EA y hagan una correcta enseñanza para la sustentabilidad (Firth y Winter, 2007). En este sentido, Yavetz et al. (2009) enfatiza que si los profesores carecen de conocimientos, habilidades o compromisos, es poco probable que tengan éxito en desarrollar en sus estudiantes la anhelada alfabetización ecológica.

Por esto, es fundamental que los profesores reciban una formación inicial ambiental que les permita satisfacer lo propuesto en el currículum nacional. Si bien en este currículum se explícita que los docentes deben realizar EA de manera transversal e interdisciplinaria, esto es difícil de llevar a cabo, pues existe la carencia de un programa integral de Educación Ambiental con lineamientos para la Educación pre-Básica, Básica, Media y Universitaria que recoja los nuevos conceptos de planificación integrada e implementación de la transversalidad, junto a metodologías que despierten la voluntad de transformar la realidad.

Es importante destacar que de acuerdo a la información recabada en esta investigación, los profesores encargados de impartir la asignatura de Ciencias Naturales no correspondieron en su totalidad a docentes con título profesional de Educación General Básica y con respecto a su mención o especialidad, éstas en su mayoría no correspondían al área de las Ciencias Naturales (gráfico 1); esto constituiría otra razón por la cual no se estaría llevando a cabo la enseñanza de la EA, puesto que al no tener una formación básica sobre temáticas del ambiente adquiridas en su formación profesional se dificultaría más aún incluirla de manera transversal en la enseñanza. Aunque, no solamente es posible desarrollar alfabetización ecológica asociado al curriculum de ciencias, sino también al de geografía (Quinn et al., 2016).

Por su parte, los criterios de la encuesta relacionados con el eje actitudes se enfocaron en determinar si los profesores estaban incluyendo actitudes y valores transversalmente 
en la enseñanza de las Ciencias Naturales. De acuerdo a los resultados, este eje superó el mínimo aceptable estimado. Esto puede atribuirse a la formación inicial de los docentes, ya que los nuevos currículos propuestos para la educación obligatoria introducen las actitudes, los valores y las normas como contenidos educativos concretos que hay que planificar, desarrollar y evaluar (Bolívar, 1992). De tal manera que enseñar y aprender actitudes es una tarea posible de realizar por los docentes en las distintas áreas de la educación. También, los diferentes desarrollos curriculares que se pueden leer en los documentos elaborados por las autoridades educativas apuntan a actitudes comunes que deben estar presentes en todas las áreas y que, por otra parte, serían deseables en cualquier ciudadano de una sociedad democrática.

Dentro de la Reforma Educativa en Chile, la EA está inserta en los OAT propuestos como los que deben contribuir significativamente al proceso de crecimiento y autoafirmación personal; a orientar la forma en que la persona se relaciona con otros seres humanos y con el mundo; a fortalecer y afianzar la formación ético-valórica; y al desarrollo del pensamiento creativo y crítico. Se tiende a pensar que los OAT deben ser enseñados solamente en el aula, pero estos pueden ser llevados a cabo mediante acciones de muy diversa índole, tanto a través del currículo manifiesto de las diferentes disciplinas como también a través de otras actividades propuestas por el establecimiento educacional (Ministerio de Educación, 2009). Por lo tanto, la transversalidad no solo interpela al currículum nacional, sino también a la cultura escolar y a los actores que forman parte de ella.

Egaña, Assael, Magendzo, Santa Cruz y Varas (2003), señala que parte de la identidad de los docentes, sobre todo los de enseñanza básica, se asocia directamente a la formación del estudiante con el desarrollo de actitudes o disposiciones valóricas, fundamentalmente ligadas al comportamiento. De esta forma, al percibir que estos fines han estado siempre en lo que se define como rol del profesor, se percibe este trabajo como presente en cada acción que se realiza, y menos como un aprendizaje que deba ser planificado para desarrollarse. Por esto, Benegas y Marcén (1995) hablan de una cierta educación moral que propone una serie de actitudes a enseñar en todas las áreas por parte de todos los educadores; produciendo ciertas modificaciones en la manera de actuar del profesorado.

Los criterios de la encuesta relacionados con el eje práctica se orientaron en determinar si los docentes están promoviendo acciones y soluciones sustentables en pro del ambiente. En este eje no se alcanzó el puntaje mínimo aceptable (gráfico 2). Una posible respuesta a lo obtenido, es que este eje constituye la dimensión más compleja dentro de los tres ejes de la EA, pues para que ésta se lleve a cabo, se necesita que el docente posea una base sólida en conocimientos y actitudes que sustenten las acciones en favor del entorno. Esta base debe ser adquirida a lo largo de la formación inicial docente, pero si esta tiene carencias influirá en su capacidad efectiva de desarrollar y ejecutar programas de EA a nivel profesional.

En Chile, no se ha profundizado en el estudio de las limitaciones o dificultades que los docentes tienen para realizar EA. En otros países, como España se ha indagado sobre éstos problemas. En este país la EA se incorporó como materia transversal de los diseños curriculares (Ley Orgánica de Ordenación General del Sistema Educativo, 1990), por ende ésta es comparable al sistema educativo Chileno. López (2001), aplicó una encuesta a profesores de niveles primarios y secundarios de la provincia de Lugo. Los resultados de este estudio dan a conocer que las preocupaciones más relevantes que tenían los docentes eran aquellas relacionadas con el tiempo u horarios y densidad de los programas; haciendo 
Estudios Pedagógicos XLIII, N³: 311-323, 2017

PRESENCIA DE UNA EDUCACIÓN AMBIENTAL BASADA EN CONOCIMIENTO, ACTITUDES Y PRÁCTICAS EN LA ENSEÑANZA DE LAS CIENCIAS NATURALES EN ESTABLECIMIENTOS MUNICIPALES DE LA CIUDAD...

difícil ubicar actividades relacionadas con EA en su práctica docente habitual. Esto no se aleja de la realidad Chilena, debido a que los docentes están sometidos a extensos horarios de trabajo y carga horaria curricular, limitando su tiempo para informarse y actualizarse sobre temas relacionados con la EA. Quizás, esto sea una de las causas por las cuales los docentes encuestados manifestaron no tener estudios y/o capacitaciones posteriores con respecto al tema; esta situación es más preocupante, ya que si consideramos los años de ejercicio profesional existe una predominancia en el rango de 0 a 10 años (gráfico 1), lo que implica que las nuevas generaciones de profesores que están realizando Ciencias Naturales presentan desmotivación y/o desinterés frente a los temas ambientales.

En la actualidad, la EA les significa a los profesores chilenos exigencias adicionales, para las cuales la mayoría no ha sido formado. A esto habría que agregar el hecho de que hay poco material pedagógico que apoyan el trabajo docente (Squella, 2000). Por lo tanto, las principales dificultades para incorporar la Educación Ambiental se relacionarían con la falta de recursos, la falta de claridad sobre cómo implementar los contenidos ambientales en el marco de la reforma educacional, la escasa prioridad política en materia de EA en el país y por último la insuficiente capacitación de profesores en el tema. Sin embargo, es necesario investigar más a fondo sobre los factores que impiden la correcta implementación de la EA en el sistema educacional chileno. Conocer estos factores permitiría evidenciar en donde debe ser puesto el énfasis para apoyar el trabajo docente en temáticas ambientales.

Es por esta razón que surge la necesidad que los docentes se formen integralmente, puesto que su labor es hacer uso de las herramientas para producir un cambio profundo en los hábitos y conductas en relación con el ambiente y así realizar la transformación del sistema escolar, para poder instalar correctamente la Reforma Educacional. El mejor método de lograr un fomento estable de la EA consiste en integrar la dimensión del ambiente en la formación inicial y permanente de los profesores, incluso también en el de otras categorías del personal de la enseñanza, en particular de aquellos que se ocupan de las diversas disciplinas relacionadas con las Ciencias Naturales y las Ciencias Sociales, así como con las asignaturas tecnológicas de nivel medio y superior. No se trata ciertamente de formar especialistas en EA, sino de dar a los educadores, cualquiera que sea su nivel, y en todos los sectores de la enseñanza, las competencias necesarias para definir los contenidos y experiencias de EA y sus problemas relacionados.

En este sentido, el Balance y Perspectivas de la Educación Ambiental en Chile e Iberoamérica (Comisión Nacional del Medio Ambiente, 2010) confirma que hoy en día es un desafío el medir la calidad de la EA en Educación General Básica, Educación Media y con mayor relevancia en las escuelas de Pedagogía, respecto de las cuales tampoco existe ninguna información que indique que los futuros docentes están recibiendo formación de calidad en cuanto a conceptos, habilidades y capacidades requeridas para ser educadores ambientales profesionales. Un antecedente que hace pensar que los futuros profesores no harán una buena EA es lo informado por Condeza (2013), quien evidencia la baja conciencia ambiental en alumnos de pedagogía, de diversas especialidades, en la ciudad de Los Ángeles.

Por ello la clave de la EA es el profesor. Si los profesores no tienen los conocimientos, las habilidades y la voluntad de incorporarla en su currículum, es muy difícil que se formen alumnos concientizados (Wilke, 1995). Los educadores de todas las disciplinas, tienen una enorme obligación ética y práctica de educar, por todos los medios pedagógicos posibles en EA. Este no es sólo un desafío de los profesores de ciencias de la naturaleza, sino de todos 
y de cada uno. En los educadores y educadoras, está parte de la responsabilidad de reparar lo destruido y de construir un mundo mejor para generaciones que nunca conoceremos (Williamson, 2005).

Como ya se ha mencionado anteriormente, para que exista una efectiva adquisición y transmisión de la EA por parte de los docentes se necesita que cada uno de sus ejes principales estén presentes de manera satisfactoria, puesto que son su núcleo fundamental y no pueden estar en desunión (Novo, 2003). Según los resultados obtenidos en esta investigación, solamente el eje actitudes es el que se encuentra presente de manera satisfactoria en la enseñanza de la EA, sin embargo, esto no es suficiente para poder afirmar que los docentes están realizando EA incluyendo sus tres ejes principales en la asignatura de Ciencias Naturales.

Quizás esto no sólo recae en la responsabilidad del educador, sino que también se deba al tratamiento que se le ha dado a las temáticas medioambientales en los Planes y Programas por parte del Ministerio de Educación, basado únicamente en los conocimientos teóricos sin darle mayor importancia a la parte actitudinal y práctica, no articulando o integrando las diferentes propuestas y finalmente no cumpliendo con el supuesto de la transversalidad en la Educación Ambiental Formal (Comisión Nacional del Medio Ambiente, 2010).

Es indudable que se han hecho avances pero éstos son insuficientes y no cubren las necesidades mínimas que tiene el país en materia de EA (Squella, 2000). Si las personas responsables de elaborar y ejecutar los programas de EA carecen de los conocimientos, habilidades y las capacidades necesarias, éstos inevitablemente serán de baja calidad.

\section{CONCLUSIONES}

Finalmente y de acuerdo a la información recopilada y analizada en esta investigación, se concluye que:

La mayoría de los docentes que realizan Ciencias Naturales en establecimientos municipalizados de la Ciudad de Los Ángeles poseen el título profesional de Educación General Básica, sin mención o especialidad en el área de Ciencias Naturales ni estudios y/o capacitaciones posteriores en Educación Ambiental.

De los tres ejes principales de la Educación Ambiental, los docentes solamente están incluyendo de manera satisfactoria el eje actitudes en la enseñanza de la Educación Ambiental en las Ciencias Naturales.

Los docentes de Educación General Básica no están realizando Ciencias Naturales contemplando de manera cohesionada los tres ejes principales de la Educación Ambiental (Conocimientos, Actitudes y Práctica).

Se rechaza la hipótesis establecida, pues el eje conocimientos no fue el predominante en la enseñanza de las Ciencias Naturales como se esperaba.

\section{REFERENCIAS BIBLIOGRÁFICAS}

Aydim, S. (2012). On the role of intrinsic value in terms of environmental education. Procedia Social and Behavioral Sciences, vol.47, 1087-1091.

Benegas, J., \& Marcén, C. (1995). La Educación Ambiental como desencadenante del cambio de 
Estudios Pedagógicos XLIII, N 3: 311-323, 2017

PRESENCIA DE UNA EDUCACIÓN AMBIENTAL BASADA EN CONOCIMIENTO, ACTITUDES Y PRÁCTICAS EN LA ENSEÑANZA DE LAS CIENCIAS NATURALES EN ESTABLECIMIENTOS MUNICIPALES DE LA CIUDAD...

actitudes ambientales. Revista Complutense de Educación, vol.6 (2),11-28.

Blum, N. (2008). Environmental education in Costa Rica: Bulding a framework for sustainable development? International Journal of Educational Development, vol.28, 348-358.

Bolivar, A. (1992). Papel del profesor en los procesos de desarrollo curricular. Revista Española de pedagogía, vol.191, 131-151.

Cabezas, M. (1997). Educación Ambiental y Lenguaje ecológico: Una propuesta didáctica para la enseñanza de la Educación Ambiental. Valladolid: Castillas Ediciones.

Çimen, O., Gökmen, A., Altunsoy, S., Ekici, G., \& Yilmaz, M. (2011). Analysis of biology candidate teachers'self efficacy beliefs on environmental education. Procedia Social and behavorial Sciences, vol.15, 2549-2553.

Comisión Nacional del Medio Ambiente. (2010). Balance y perspectivas de la Educación Ambiental en Chile e Iberoamérica: Reflexiones sobre la educación ambiental en Chile. Chile: Gráfica Metropolitana.

Condeza, K. (2013). Estudio de la relación entre el grado de conciencia ambiental en docentes y estudiantes de pedagogía y su área de especialidad: ¿puede un docente hacer Educación Ambiental sin conciencia ambiental? Seminario de Título para optar el título de Profesor de Ciencias Naturales y Biología. Los Ángeles: Universidad de Concepción.

Egaña, L., Assael, J., Magendzo, A., Santa Cruz, E., \& Varas, R. (2003). Reforma Educativa y Objetivos Fundamentales Transversales. Los dilemas de la innovación. Santiago: Programa interdisciplinario de Investigación en Educación.

Esa, N. (2010). Environmental knowledge, attitude and practices of student teachers. International Research in Geographical and Environmental Education, vol.19 (1), 39-50.

Ferreira, Y., Ryan, L., \& Tilbury, D. (2007). Mainstreaming education for sustainable development in initial teacher education in Australia: a review of existing profesional development models. Journal of Education for Teaching, vol.33 (2), 225-239.

Firth, R., \& Winter, C. (2007). Constructing education for sustainable development the secondary school geography curriculum and initial teacher training. Environmental Education Research, vol.13 (5), 599-619.

George, D., \& Mallery, P. (2003). SPSS for Windows: Step by step. A simple guide and reference 11.0 update. Boston: Allyn and Bacon.

González, E. (2003). Educación para la ciudadanía ambiental. Interciencia Caracas Venezuela, vol.28 (10), 611-615.

Hernández, R., Fernández, C., \& Baptista, P. (2010). Metodología de Investigación. (5ºdición). México: McGraw-Hill.

Kennelly, J., Taylor, N., \& Maxwell, T. (2008). A student teacher's personal pathway to education for sustainability. Australian Journal of Education, vol.24, 23-33.

Lee, C., \& Williams, M. (2001). Researching Environmental Education in the school curriculum: an introduction for students and teachers researches. International Research in Geographical and Environmental Education, vol.10 (3), 218-244.

Ley Orgánica de Ordenación General del Sistema Educativo (1990). Recuperado el 15 de Agosto de 2014 desde https://www.boe.es/boe/dias/1990/10/04/pdfs/A28927-28942.pdf.

López, R. (2001) Qué Problemas preocupan principalmente al profesorado para llevar a cabo Educación Ambiental. Revista de currículum y formación del profesorado, vol.5 (2), 1-9.

MC Pherson, S., \& Hernández, P. (2001). La Educación Ambiental en la enseñanza de las Ciencias. Recuperado el 10 de Marzo 2014 desde http://www.bio-nica.info/biblioteca/McPhersonEducacionAmbiental.pdf.

Ministerio de Educación. (2012). Programa de estudio de Enseñanza Básica, Ciencias Naturales. Santiago: Ministerio de Educación.

Ministerio de Educación. (2009). Contenidos Mínimos Obligatorios y Objetivos Fundamentales Transversales para Educación General Básica. Santiago: Ministerio de Educación. 
Novo, M. (2003). La educación ambiental. Bases éticas, conceptuales y metodológicas. Madrid: Editorial Universitarias.

Quinn, F., Castera, J., \& Clement, P. (2016). Teachers'conceptions of the environmental: anthropocentrism, non- anthropocentrism, anthropomorphism and the place of nature. Environmental Education Research, Vol.22 (6), 893-917.

Shymansky, J., \& Kyle, W. (1992). Establishing a research agenda: Critical issues of science curriculum reform. Journal of Research in Science Teaching, vol.29 (8), 749-778.

Squella, M. (2000). La educación ambiental en Chile. Un estudio exploratorio. Münster: Editorial LIT Verlag Berlín.

Sureda-Negre, J., Oliver-Trobat, M., Catalán-Fernández,A., \& Comas-Forgas, R. 2014. Environmental education for sustainability in the curriculum of primary teacher training in Spain. International Research in Geographical and Environmental Education, vol.23 (4), 281-293.

Tuncer, G., Tekkaya, C., Sungur, S., Cakiroglu, J., Ertepinar, H., \& Kaplowitz, M. 2009. Assessing pre-service teachers' environmental literacy in Turkey as a mean to develop teacher education programs. International Journal of Educational Development, vol.29 (4), 426-436.

Organización de las Naciones Unidas para la Educación, la Ciencia y la Cultura. (1975). Seminario Internacional de Educación Ambiental. Programa internacional de Educación Ambiental. Belgrado: UNESCO.

Vidal, J. (2005). Manual de trabajo de campo en la encuesta. Madrid: Centro de Investigaciones Sociológicas.

Welch, S., \& Comer, J. (1988). Quantitative methods for public administration: techniques and applications. Virginia: Universidad de Virginia.

Wilke, R. (1995). Programa de Educación Ambiental para profesores e inspectores de ciencias sociales de Enseñanza Media. Bilbao: Los libros de la Catarata.

Williamson, G. (2005) Territorios de aprendizajes interculturales: transitando a una nueva praxis pedagógica. Revista Pensamiento Educativo, vol.37, 163-181.

Yavetz, B., Goldman, D., \& Pe'er, S. 2009. Environmental literacy of pre-service teachers in Israel: a comparison between students at the onset and end their studies. Environmental Education Research, vol.15 (4), 393-415. 
